\title{
Water Pollution Indicator using Arduino Uno
}

\author{
Prof. SUMATHI.K \\ SNS College of Technology, Coimbatore, India \\ Prof. CHRISTINA DALLY.E \\ SNS College of Technology, Coimbatore, India \\ Prof.ASHWINI G.V \\ SNS College of Technology, Coimbatore, India \\ SAIGOKUL.s \\ SNS College of Technology, Coimbatore, India
}

\begin{abstract}
Immaculate drinking water is critical for the wellbeing and prosperity of all people and creatures because water play major role in all living beings and most danger disease are caused by water and it is our duty to provide clean and safe water and also to monitor the pollution level in water it is additionally essential for farming utilisation for good product yielding and natural way of life linkage wellbeing issues.An ease and comprehensive methodology is micro controller sensor based system continuous observing and pollution recognition for both drinking and non-drinking water dissemination frameworks and in addition for customer locales
\end{abstract}

\section{I.INTRODUCTION}

Clean drinking water is critical resource, important for the health and well-being of all humans. several experimental studies indicate need for continues online water monitoring efficient patio-temporal resolution and demonstrate that the conventional reagent-based water quality methods fail to satisfy this requirements due to higher labour and operational cost it is critical for accurate real-time water quality observing frameworks than the current lab based techniques, which are too ease back to create operational reaction and don't give a level of general wellbeing security continuously conventional strategies for water quality checking and control include the manual accumulation of water test at different areas and at diverse time trailed by research centre scientific procedures keeping in mind the end goal to portray the water quality the main conclusion was that many of the chemical and biological contaminants used have an effort on many water parameters monitored including Turbidity (TU), oxidation Reduction Potential (ORP),Electrical conductivity(EC) and $\mathrm{Ph}$. Thus it is easy to monitor and infer the water quality by detecting the changes in such parameters.

\section{II.EXISTING SYSTEM}

In existing system we use a 32-Bit of microcontroller The main goal is the design and development of low cost system that can be used at the premises of consumers. This system can also be used in customer oriented manner to continuously monitor qualitative water parameters and fuse multi parametric sensor data for assessing the water quality. A 32-bit Microcontroller and associated sensor network can detect water contamination continuously according to water conductivity and temperature. Depends on these parameters microcontroller computes the other related contaminants and transmits same data to consumers and some authorities by using GSM module. A user/consumer can view the presented data regarding quality of water and it will be used accordingly. The below shows a functional block diagram proposed the low cost system for both potable and non-potable water comprising of multi sensor network and 32-bit microcontrollerdevelopment board, GPRS/GSM module etc.

A preliminary version of this article has appeared. In this article, we present an improved hardware platform, develop a new advanced contamination event detection algorithm and provide an experimental evaluation and validation of system and event detection algorithms in the presence of Real microbiological and chemical contamination events. In addition, several water monitoring micro systems (sensor nodes) have been developed for large scale water monitoring based on wireless sensor networks (WSNs) technology. A sensor hub is created for checking saltiness in ground water and the water temperature in surface waters. We have built up a WSN and a vitality gathering framework (in view of a sun powered board) to screen nitrate, ammonium and chloride levels in 
streams and lakes. Vitality reaping systems alongside hibernation techniques assume a critical part in expanding the lifetime of sensor hubs. A study on vitality collecting for WSNs is given. a self-governing vessel furnished with water sensors is proposed to gather

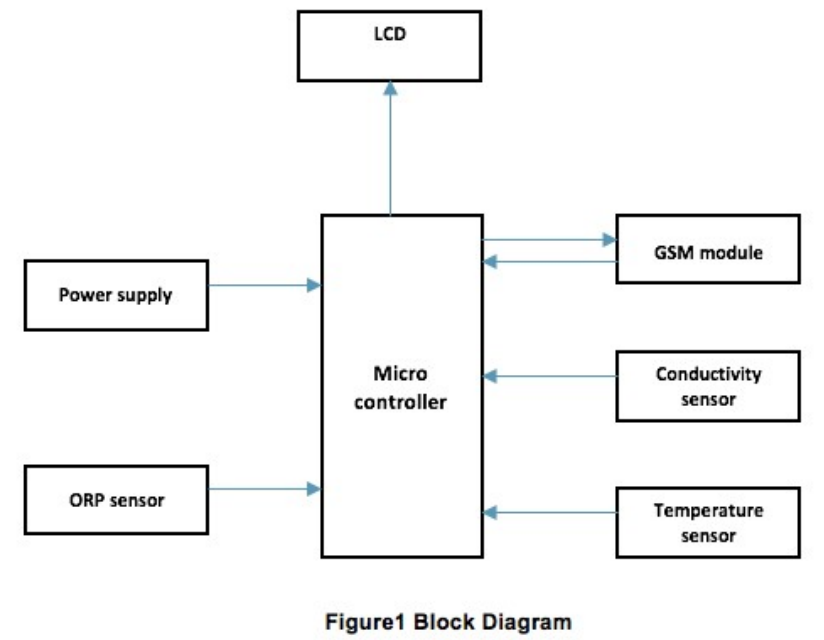

III.PROPOSED SYSTEM

In proposed system we use Arduino Uno instead of an32-bit microcontroller The Arduino Uno is a microcontrollerboard based on the ATmega328. It has 14 digital input/output pins (of which 6 can be used as PWM outputs), 6 analogue inputs, a $16 \mathrm{MHz}$ ceramic resonator, a USB connection, a power jack, an ICSP header, and a reset button. It contains everything needed to support the microcontroller; simply connect it to a computer with a USB cable or power it with a AC-to-DC adapter or battery to get started. The Uno differs from all preceding boards in that it does not use the FTDI USB-to-serial driver chip. Instead, it features the Atmega16U2 (Atmega8U2 up to version R2) programmed as a USB-to-serial converter.

The board has the following new features:

pinout: added SDA and SCL pins that are near to the AREF pin and two other new pins placed near to the RESET pin, the IOREF that allow the shields to adapt to the voltage provided from the board. In future, shields will be compatible both with the board that use the AVR, which operate with 5V and with the Arduino Due that operate with $3.3 \mathrm{~V}$. The second one is a not connected pin, that is reserved for future purposes.

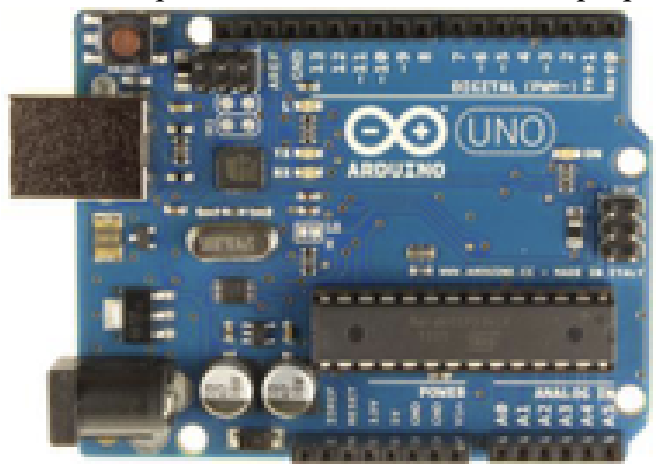

Figure2. Arduino Uno

Stronger RESET circuit.

At mega $16 \mathrm{U} 2$ replace the $8 \mathrm{U} 2$. 
"Uno" means one in Italian and is named to mark the upcoming release of Arduino 1.0.

\begin{tabular}{|c|c|c|}
\hline \multicolumn{3}{|l|}{ Microcontroller } \\
\hline Operating Voltage & & \\
\hline \multicolumn{3}{|c|}{ Input Voltage (recommended) $7-12 \mathrm{~V}$} \\
\hline \multicolumn{3}{|c|}{ Input Voltage (limits) $\quad 6-20 \mathrm{~V}$} \\
\hline \multicolumn{3}{|c|}{ Digital I/O Pins $\quad 14$ (of which 6 provides PWM output) } \\
\hline \multicolumn{3}{|c|}{ Analog Input Pins 6} \\
\hline \multirow{2}{*}{\multicolumn{3}{|c|}{$\begin{array}{l}\text { DC Current per I/O Pin } \\
\text { DC Current for } 3.3 \mathrm{~V} 6\end{array}$}} \\
\hline & & \\
\hline Pin & & \\
\hline Flash Memory & $32 \mathrm{~KB}$ & \\
\hline SRAM & & 2KB (ATmega328) \\
\hline EEPROM & & 1KB (ATmega328) \\
\hline Clock speed & & $16 \mathrm{MHz}$ \\
\hline
\end{tabular}

IV.POWER

The Arduino Uno can be powered via the USB connection or with an external power supply. The power source is selected automatically. External (non-USB) power can come either from an AC-to-DC adapter (wall-wart) or battery. The adapter can be connected by plugging a $2.1 \mathrm{~mm}$ centre-positive plug into the board's power jack. Leads from a battery can be inserted in the Ground and Vin pin headers of the power connector. The board can operate on an external supply of 6 to 20 volts.If using more than $12 \mathrm{~V}$, the voltage regulator may overheat and damage the board. The recommended range is 7 to 12 volts.

\subsection{THE POWER PINS ARE AS FOLLOWS:}

VIN. The input voltage to the Arduino board when it's using an external power source (as opposed to 5 volts from the USB connection or other regulated power source). You can supply voltage through this pin, or, if supplying voltage via the power jack, access it through this pin.

$5 \mathrm{~V}$. This pin outputs a regulated $5 \mathrm{~V}$ from the regulator on the board. The board can be supplied with power either from the DC power jack (7 - 12V), the USB connector (5V), or the VIN pin of the board (7-12V). Supplying voltage via the $5 \mathrm{~V}$ or $3.3 \mathrm{~V}$ pins bypasses the regulator, and can damage your board. We don't advise it.

3V3. A 3.3-volt supply generated by the on-board regulator. Maximum current draw is $50 \mathrm{~mA}$.

GND. Ground pins.

MEMORY The ATmega328 has $32 \mathrm{~KB}$ (with $0.5 \mathrm{~KB}$ used for the bootloader). It also has $2 \mathrm{~KB}$ of SRAM and 1 KB of EEPROM (which can be read and written with the EEPROM library).

\section{V.INPUT AND OUTPUT}

Each of the 14 digital pins on the Uno can be used as an input or output, using pin Mode(),digital Write(), and digital Read() functions. They operate at 5 volts. Each pin can provide or receive a maximum of $40 \mathrm{~mA}$ and has an internal pull- up resistor (disconnected by default) of 20-50 ohms. In addition, some pins have

Serial: 0 (RX) and 1 (TX). Used to receive (RX) and transmit (TX) TTL serial data. These pins are connected to the corresponding pins of the ATmega8U2 USB-to-TTL Serial chip.

External Interrupts: 2 and 3. These pins can be configured to trigger an interrupt on a low value, a rising or falling edge, or a change in value. See the attach Interrupt() function for details.

PWM: 3, 5, 6, 9, 10, and 11. Provide 8-bit PWM output with the analogue Write() function.

SPI: 10 (SS), 11 (MOSI), 12 (MISO), 13 (SCK). These pins support SPI communication using the SPI library.

LED: 13 . There is a built-in LED connected to digital pin 13. When the pin is HIGH value, the LED is on, when the 
pin is LOW, it's off. The Uno has 6 analogue inputs, labelled A0 through A5, each of which provide 10 bits of resolution (i.e.1024 different values). By default, they measure from ground to 5 volts, though is it possible to change the upper end of their range using the AREF pin and the analogue Reference. Additionally, some pins have specialized functionality:

TWI: A4 or SDA pin and A5 or SCL pin. Support TWI communication using the Wire library. There are a couple of other pins on the board:

AREF. Reference voltage for the analogue inputs. Used with analogue Reference ().

Reset. Bring this line LOW to reset the microcontroller. Typically used to add a reset button to shields which block the one.

\section{VI.COMMUNICATION}

The Arduino Uno has a number of facilities for communicating with a computer, another Arduino, or other microcontrollers. The ATmega328 provides UART TTL (5V) serial communication, which is available on digital pins 0 (RX) and 1 (TX). An ATmega16U2 on the board channels this serial communication over USB and appears as a virtual com port to software on the computer. The ' $16 \mathrm{U} 2$ firmware uses the standard USB COM drivers, and no external driver is needed. However, on Windows, a in file is required. The Arduino software includes a serial monitor which allows simple textual data to be sent to and from the Arduino board. The RX and TX LEDs on the board will flash when data is being transmitted via the USB-to-serial chip and USB connection to the computer (but not for serial communication on pins 0 and 1 ).

A Software Serial library allows for serial communication on any of the Uno's digital pins. The A Tmega328 also supports I2C (TWI) and SPI communication. The Arduino software includes a Wire library to simplify use of the I2C bus; see the documentation for details. For SPI communication, use the SPI library.

\section{VII.PROGRAMMING}

The Arduino Uno can be programmed with the Arduino software (download). Select "Arduino Uno from the Tools > Board menu (according to the microcontroller on your board). The ATmega328 on the Arduino Uno comes preboned with a bootloader that allows you to upload new code to it without the use of an external hardware programmer. It communicates using the original STK500 protocol (reference, C header files). You can also bypass the bootloader and program the microcontroller through the ICSP (In-Circuit Serial Programming) header; see these instructions for details. The ATmega16U2 (or $8 \mathrm{U} 2$ in the rev1 and rev2 boards) firmware source code is available. The ATmega16U2/8U2 is loaded with a DFU bootloader, which can be activated by:

On Rev1 boards: connecting the solder jumper on the back of the board (near the map of Italy) and then resetting the $8 \mathrm{U} 2$.

On Rev2 or later boards: there is a resistor that pulling the $8 \mathrm{U} 2 / 16 \mathrm{U} 2 \mathrm{HWB}$ line to ground, making it easier to put into DFU mode.

You can then use Atmel's FLIP software (Windows) or the DFU programmer (Mac OS X and Linux) to load a new firmware.

\section{VIII.AUTOMATIC (SOFTWARE) RESET}

Rather than requiring a physical press of the reset button before an upload, the Arduino Uno is designed in a way that allows it to be reset by software running on a connected computer. One of the hardware flow control lines (DTR) of the A Tmega8U2/16U2 is connected to the reset line of the ATmega328 via a 100 Nano farad capacitor. When this line is asserted (taken low), the reset line drops long enough to reset the chip. The Arduino software uses this capability to allow you to upload code by simply pressing the upload button in the Arduino environment. This means that the bootloader can have a shorter timeout, as the lowering of DTR can be well-coordinated with the start of the upload. 
This setup has other implications. When the Uno is connected to either a computer running Mac OS X or Linux, it resets each time a connection is made to it from software (via USB). For the following half second or so, the bootloader is running on the Uno. While it is programmed to ignore malformed bootloader and program the microcontroller through the ICSP (In-Circuit Serial Programming) header; see these instructions for details. The ATmega16U2 (or 8U2 in the rev1 and rev2 boards) firmware source code is available. The ATmega16U2/8U2 is loaded with a DFU bootloader, which can be activated by

On Rev1 boards: connecting the solder jumper on the back of the board (near the map of Italy) and then resetting the $8 \mathrm{U} 2$.

On Rev2 or later boards: there is a resistor that pulling the 8U2/16U2 HWB line to ground, making it easier to put into DFU mode.

You can then use Atmel's FLIP software (Windows) or the DFU programmer (Mac OS X and Linux) to load a new firmware. Or you can use the ISP header with an external programmer (overwriting the DFU bootloader).

\section{IX.AUTOMATIC RESET}

Rather than requiring a physical press of the reset button before an upload, the Arduino Uno is designed in a way that allows it to be reset by software running on a connected computer. One of the hardware flow control lines (DTR) of the A Tmega8U2/16U2 is connected to the reset line of the ATmega328 via a 100 Nano farad capacitor. When this line is asserted (taken low), the reset line drops long enough to reset the chip. The Arduino software uses this capability to allow you to upload code by simply pressing the upload button in the Arduino environment. This means that the bootloader can have a shorter timeout, as the lowering of DTR can be well-coordinated with the start of the upload. This setup has other implications. When the Uno is connected to either a computer running Mac OS X or Linux, it resets each time a connection is made to it from software (via USB). For the following half second or so, the bootloader is running on the Uno. While it is programmed to ignore malformed data (i.e. anything besides an upload of new code), it will intercept the first few bytes of data sent to the board after a connection is opened. If a sketch running on the board receives one-time configuration or other data when it first starts, make sure that the software with which it communicates waits a second after opening the connection and before sending this data. The Uno contains a trace that can be cut to disable the auto-reset. The pads on either side of the trace can be soldered together to re-enable it. It's labelled "RESET-EN". You may also be able to disable the auto-reset by connecting a 110-ohm resistor from $5 \mathrm{~V}$ to the reset line; see this forum thread for details. The Arduino Uno has a resettable poly fuse that protects your computer's USB ports from shorts and overcurrent. Although most computers provide their own internal protection, the fuse provides an extra layer of protection. If more than $500 \mathrm{~mA}$ is applied to the USB port, the fuse will automatically break the connection until the short or overload is removed.

\section{X.PHYSICAL CHARACTERISTICS}

The maximum length and width of the Uno PCB are 2.7 and 2.1 inches respectively, with the USB connector and power jack extending beyond the former dimension. Four screw holes allow the board to be attached to a surface or case. Note that the distance between digital pins 7 and 8 is 160 mil(0.16"), not an even multiple of the 100 mil spacing of the other pins.

GSM

GSM/GPRS module is used to establish communication between a computer and a GSM-GPRS system. Global System for Mobile communication (GSM) is an architecture used for mobile communication in most of the countries. Global Packet Radio Service (GPRS) is an extension of GSM that enables higher data transmission rate. GSM/GPRS module consists of a GSM/GPRS modem assembled together with power supply circuit and communication interfaces (like RS-232, USB

, etc.) 


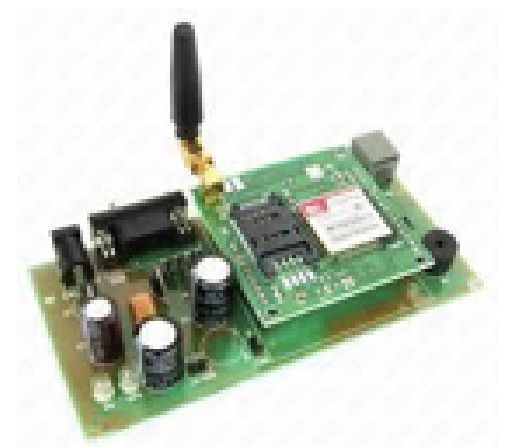

Fgure3: GSM Module SENSOR

The $\mathrm{pH}$ of a solution indicates how acidic or basic (alkaline) it is. The $\mathrm{pH}$ term translates the values of the hydrogen ion concentration which ordinarily ranges between about 1 and $10 \mathrm{x}-14$ gram-equivalents per litre - into numbers between 0 and 14 . On the $\mathrm{pH}$ scale a very acidic solution has a low $\mathrm{pH}$ value such as 0,1 , or 2 (which corresponds to a large concentration of hydrogen ions; $10 \times 0,10 \times-1$, or $10 \times-2$ gram-equivalents per litre) while a very basic solution has a high $\mathrm{pH}$ value, such as 12,13 , or 14 which corresponds to a small number of hydrogen ions $(10 \times-12,10 \times-13$, or $10 \times-14$ gram-equivalents per litre).A neutral solution such as water has a $\mathrm{pH}$ of approximately 7.A pH measurement loop is made up of three components, the $\mathrm{pH}$ sensor, which includes a measuring electrode a reference electrode, and a temperature sensor; a preamplifier; and an analyser or transmitter. A pH measurement loop is essentially a battery where the positive terminal is the measuring electrode and the negative terminal is the reference electrode. The measuring electrode, which is sensitive to the hydrogen ion, develops a potential (voltage) directly related to the hydrogen ion concentration of the solution. The reference electrode provides a potential against which the measuring electrode can be compared.

\subsection{TURBIDITY SENSOR}

The turbidity sensor detects water quality by measuring the levels of turbidity. It uses light to detect suspended particles in water by measuring the light transmittance and scattering rate, which changes with the amount of total suspended solids (TSS) in water. As the TTS increases, the liquid turbidity level increases.Turbidity sensors are used to measure water quality in rivers and streams, wastewater and effluent measurements, control instrumentation for settling ponds,sediment transport research and laboratory measurements.

This sensor provides analogue and digital signal output modes.

\subsection{TEMPERATURE SENSORS}

Temperature is the most often-measured environmental quantity. This might be expected since most physical, electronic, chemical, mechanical, and biological systems are affected by temperature. Certain chemical reactions, biological processes, and even electronic circuits perform best within limited temperature ranges. 


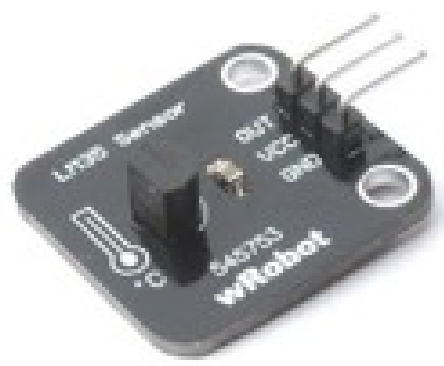

Figure4 Temperature Sensor

Temperature is one of the most commonly measured variables and it is therefore not surprising that there are many ways of sensing it. Temperature sensing can be done either through direct contact with the heating source, or remotely, without direct contact with the source using radiated energy instead. There are a wide variety of temperature sensors on the market today, including Thermocouples, Resistance Temperature Detectors (RTDs), Thermistors, Infrared, and Semiconductor Sensors. The LM35 series are precision integrated-circuit temperature sensors, whose output voltage is linearly proportional to the Celsius temperature. The LM35 is operates at $-55^{\circ}$ to $+120^{\circ} \mathrm{C}$. The basic centigrade temperature sensor $\left(+2{ }^{\circ} \mathrm{C}\right.$ to $\left.+150^{\circ} \mathrm{C}\right)$. The LM35 can be connected easily in the same way as other integrated circuit temperature sensors. It can be stuck or established to a surface and its temperature will be within around the range of $0.01{ }^{\circ} \mathrm{C}$ of the surface temperature. This presumes that the ambient air temperature is just about the same as the surface temperature; if the air temperature were much higher or lower than the surface temperature, the actual temperature of the LM35 die would be at an intermediate temperature between the surface temperature and the air temperature.

\subsection{FLOW SENSOR}

The sensors are solidly constructed and provide a digital pulse each time an amount of water pass through the pipe. The output can easily connect to a micro controller for monitoring water usage and calculating the amount of water remaining in the tank. This sensor are ideal for use in water conservation system, storage tanks, water recycling home applications, irrigation system. A wide supply voltage can be connected to the unit. The unit is constructed of long life polymer and suitable for outdoor mounting.

\subsection{SOLENOID VALVE}

A solenoid valve is an electromechanically operated valve. The valve is controlled by an electric current through a solenoid: in the case of a two-port valve the flow is switched on or off; in the case of a three-port valve, the outflow is switched between the two outlet ports. Multiple solenoid valves can be placed together on a manifold. Solenoid valves are the most frequently used control elements in fluidics. Their tasks are to shut off, release, dose, distribute or mix fluids. They are found in many application areas. Solenoids offer fast and safe switching, high reliability, long service life, good medium compatibility of the materials used, low control power and compact design. 


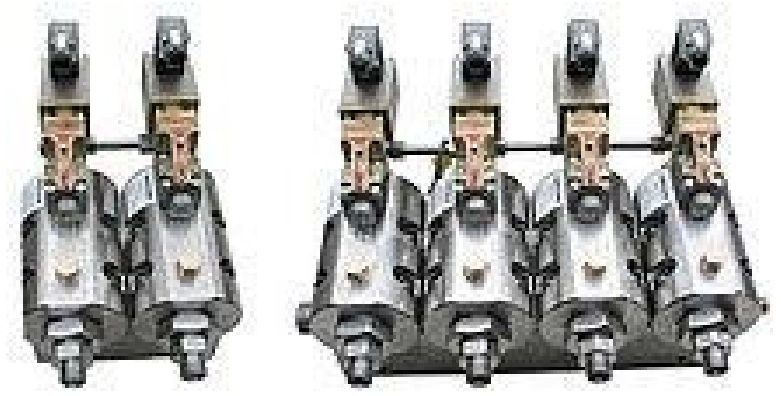

Figure5. Solenoid valves

$10.5 L C D$

A liquid crystal display (commonly abbreviated LCD) is a thin, flat display device made up of any number of colour or monochrome pixels arrayed in front of a light source or reflector. It is often utilized in battery- powered electronic devices because it uses very small amounts of electric power. Each pixel of an LCD typically consists of a layer of molecules aligned between two transparent electrodes, and two polarizing filters, the axes of transmission of which are (in most of the cases) perpendicular to each other. With no liquid crystal between the polarizing filters, light passing through the first filter would be blocked by the second (crossed) polarizer.

The surfaces of the electrodes that are in contact with the liquid crystal material are treated so as to align the liquid crystal molecules in a particular direction. This treatment typically consists of a thin polymer layer that is uni directionally rubbed using, for example, a cloth. The direction of the liquid crystal alignment is then defined by the direction of rubbing.

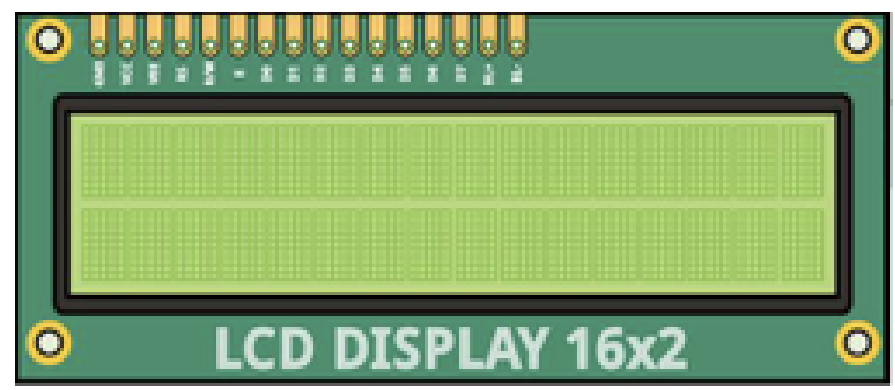

Figure5 LCD Display

\subsection{TRANSFORMER}

A transformer is a device that transfers electrical energy from one circuit to another through inductively coupled wires. A changing current in the first circuit (the primary) creates a changing magnetic field; in turn, this magnetic field induces a changing voltage in the second circuit (the secondary). By adding a load to the secondary circuit, one can make current flow in the transformer, thus transferring energy from one circuit to the other. The transformer is based on two principles: first, that an electric current can produce a magnetic field (electromagnetism) and, second, that a changing magnetic field within a coil of wire induces a voltage across the ends of the coil (electromagnetic induction). By changing the current in the primary coil, one changes the strength of its magnetic field; since the secondary coil is wrapped around the same magnetic field, a voltage is induced across the secondary. 


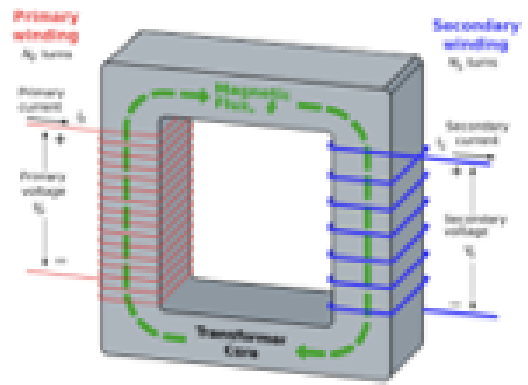

Figur6.An ideal step-down transformer showing magnetic flux in the core

\subsection{POWER SUPPLY}

A power supply (sometimes known as a power supply unit or PSU) is a device or system that supplies electrical or other types of energy to an output load or group of loads. The term is most commonly applied to electrical energy supplies, less often to mechanical ones, and rarely to others. The transformer steps up or steps down the input line voltage and isolates the power supply from the power line.

\subsection{RELAY:}

Relays are components which allow a low-power circuit to switch a relatively high current on and off, or to control signals that must be electrically isolated from the controlling circuit itself.

To make a relay operate, you have to pass a suitable pull-in and holding current (DC) through its energizing coil. And generally relay coils are designed to operate from a particular supply voltage - often $12 \mathrm{~V}$ or $5 \mathrm{~V}$, in the case of many of the small relays used for electronics work. In each case the coil has a resistance which will draw the right pull-in and holding currents when it is connected to that supply voltage. So the basic idea is to choose a relay with a coil designed to operate from the supply voltage you're using for your control circuit (and with contacts capable of switching the currents you want to control), and then provide a suitable relay driver circuit so that your low-power circuitry can control the current through the relay coil. Typically this will be somewhere between $25 \mathrm{~mA}$ and $70 \mathrm{~mA}$.

\section{XI.SOFTWARE DESCRIPTION}

Proteus developed by Lab centre Electronics, is a software with which you can easily generate schematic captures, develop PCB and simulate microcontroller. It provides a powerful working environment. The user can design different electronic circuits with all necessary components easily accessible like signal generators, power supply, simple resistor and a different microcontroller or microprocessor

VCM (Virtual System Modelling) feature allows the real time design simulation. ARES (Advanced Routing and Editing Software) is another powerful feature that permits you to route or edit the different components which are used for producing printed circuits.

\subsection{FEATURES OF PROTEUS 8}

Easy to use tool,

Simple but effective interface,

Circuit designing and schematic making made easy,

Provides a powerful working environment,

Real time design simulation with VSW,

Can route and edit different components using ARES feature. 


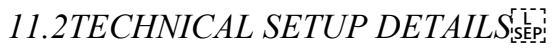

Software full name: Proteus 8.0

Setup name: Proteus_8_Professional_Setup.exe Full Setup size: 162 MB

Setup type: Off line installer/full space standalone setup

\subsection{SYSTEM REQUIREMENTS FOR PROTEUS8}

Compatibility Architecture:32 Bit/64 Bit

Operating System : Windows XP/vista/7/8

Memory(RAM):256 MB

Hard disk space: $200 \mathrm{MB}$

Processor : 233 MHZ Processor or higher

\section{XII.RESULT}

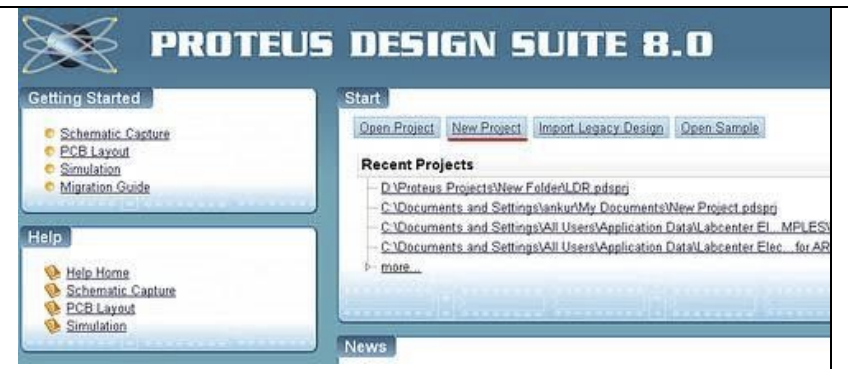

Figure7. Proteus Software

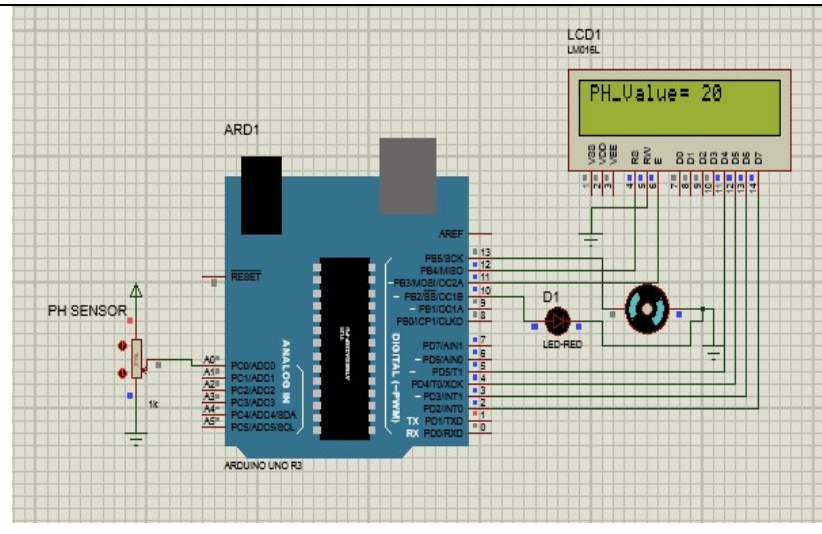

Figure8. Hardware Simulation 


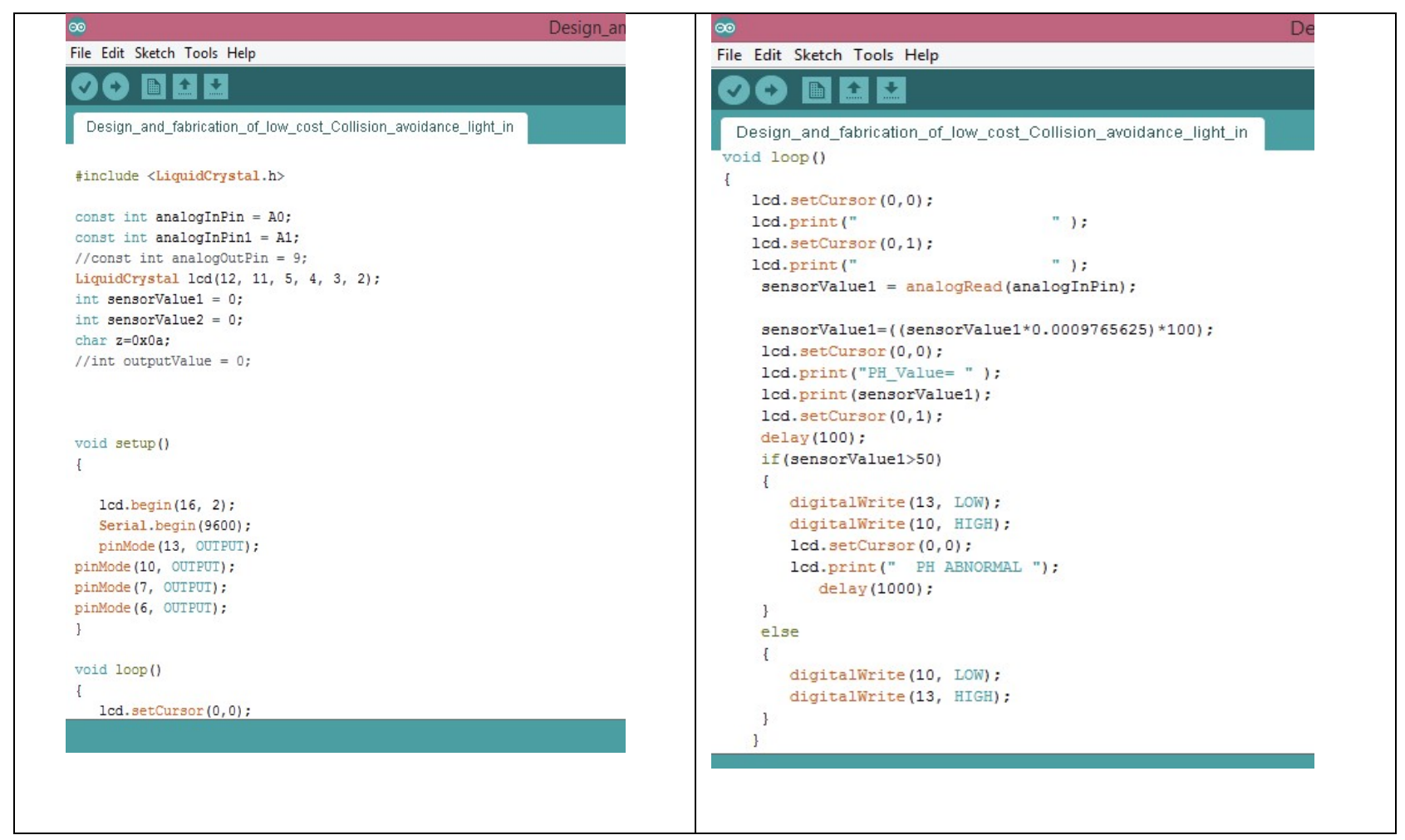

\section{REFERENCES}

[1] A. Aisopou, I. Stoianov, N. Graham, In-pipe water quality monitoring in water supply system under steady and unsteady state flow conditions: A quantitative assessment, Water Research, 46(1), pp. 235-246, 2012

[2] S. Panguluri, G. Meiners, J. Hall, J.G. Szabo, Distribution System Water Quality Monitoring: Sensor Technology Evaluation Methodology and Results, U.S. Environmental Protection Agency, EPA/600/R-09/076, 2009

[3] J. Hall, A.D. Zaffiro, R.B. Marx, P.C. Kefauver, E.R. Krishnan, R.C. Haught, and J.G. Herrmann, On-line Water Quality Parameters as Indicators of Distribution System Contamination, Journal AWWA, 2007

[4] Hach HST, Guardian Blue Early Warning System Brochure. [5] JMAR, Bio Sentry Contamination Warning System Technical Overview.

[5] World Health Organization Guidelines for drinking-water quality, fourth edition, 2011

[6] European Communities Drinking Water Regulations (No.2), 2007

[7] U.S. Environmental Protection Agency 2012 Edition of the Drinking Water Standards and Health Advisories, EPA 822-S-12-001

[8] C.N.Has, M.A. Meyer, and M.S. Paller, Microbial alterations in water distribution systems and their relationship to physical-chemical characteristics, in Journal of American Water Works Association, 1983

[9] K.N. Power and L.A. Nagy, Relationship between bacterial regrowth and some physical and chemical1 '........ parameters within Sydney's drinking water distribution system, in Water Research, 1999

[10] J. B. Cook, J. F. Byrne, R. C. Da men and E. A. Roehl, Distribution System Monitoring Research at Charleston Water System, in 8th Annual Water Distribution Systems Analysis Symposium, 2006

[11] Y.J. Yang, R.C Haught and J.A Goodrich, Real-time contaminant detection and classification in a drinking water pipe using conventional water quality sensors: Techniques and experimental results, in Journal of Environmental Management, 2009

[12] C.C. Anastasiou, P. Grafias, T.P. Lambrou, A. Kalli and C. Onisiphorou, Use of holding tanks and their effect on the quality of potable water in households; the case study of Cyprus, in PRE XI, 2012

[13] T.P. Lambrou, $\quad$ A $\quad$ Technical Re

ort for Water Qualitative and Quantitative Sensors, University of Cyprus, 2011

[14] A. Whelan and F.Regan, Antifouling strategies for marine and riverine sensors, Journal of Environmental Monitoring, 2006

[15] SensoreX Corp Flat Surface Operating Principles.

[16] T.P. Lambrou, C.C. Anastasiou , C.G. Panayiotou, A Nephelometric Turbidity System for Monitoring Residential Drinking Water Quality, in Sensor Networks Applications, Experimentation and Logistics, 2009 
International Journal of Innovations in Engineering and Technology (IJIET) http://dx.doi.org/10.21172/ijiet.92.05 